\title{
Type-D Personality Can Predict Suicidality in Patients with Major Depressive Disorder
}

\author{
Young-Min Park ${ }^{\circledR}$, Young-Hoon $\mathrm{Ko}^{2}$, Moon-Soo Lee ${ }^{2}$, Heon-Jeong Lee ${ }^{2}$, and Leen $\mathrm{Kim}^{2}$ \\ ${ }^{1}$ Department of Psychiatry, Ilsan Paik Hospital, Inje University College of Medicine, Goyang, Republic of Korea \\ ${ }^{2}$ Department of Psychiatry, Korea University College of Medicine, Seoul, Republic of Korea
}

Objective This study investigated the putative association between type-D personality and suicidality, including the history of suicide attempt and suicidal ideation in patients with major depressive disorder (MDD).

Methods Eighty-six outpatients aged between 18 and 65 years with MDD were recruited for this study from Ilsan Paik Hospital. The cohort was stratified into two subgroups according to the presence of type-D personality and history of suicide attempt (yes vs. no). Depression severity was evaluated using the Hamilton Depression Rating Scale. The type-D Personality Scale-14 (DS-14), the Beck Hopelessness Scale (BHS), the Barratt Impulsiveness Scale (BIS), the Hamilton Anxiety Scale, and the Beck Scale for Suicidal Ideation (BSS) were also applied.

Results The total BSS, BHS, and BIS scores were higher for the group with type-D personality than for the group without this personality ( $\mathrm{p}=0.004,0.01$, and 0.003 , respectively). In addition, the total scores for the BSS, BHS, and social inhibition (SI; subscale of DS-14) were higher for the group with a history of suicide attempt than for the group without this history ( $\mathrm{p}=0.0000004,0.003$, and 0.033 , respectively). There were positive correlations between the total DS- 14 score and the total BSS, BHS, and BIS scores ( $r=0.413$ and $p=0.000077$, $r=0.404$ and $\mathrm{p}=0.00012$, and $\mathrm{r}=0.245$ and $\mathrm{p}=0.024$, respectively).

Conclusion Depressed patients with type-D personality are more vulnerable to suicidality than those without type-D personality, even when the MDD severity is identical. In addition, the SI score was higher in patients with a history of suicide attempt than in those without this history.

Psychiatry Investig 2014;11(3):232-236

Key Words Suicide, Type-D personality, Major depressive disorder, DS-14.

\section{INTRODUCTION}

The type-D Personality Scale-14 (DS-14) was developed in Europe because the relationship between cardiovascular disease and type-A personality had not been clarified. ${ }^{1}$ The DS14 comprises the subscales of negative affectivity (NA) and social inhibition (SI). NA refers to the tendency to experience negative emotions, while SI refers to the tendency to inhibit the expression of emotions and behaviors. ${ }^{2}$ Individuals scoring greater than the cutoff value for both subscales are classified as having a type-D personality, ${ }^{3}$ which is associated with

Received: November 26, 2013 Revised: January 1, 2014 Accepted: January 11, 2014 Available online: July 9, 2014

$\triangle$ Correspondence: Young-Min Park, MD, PhD

Department of Psychiatry, Ilsan Paik Hospital, Inje University College of Medicine, 170 Juhwa-ro, Ilsansu-gu, Goyang 411-706, Republic of Korea

Tel: +82-31-910-7260, Fax: +82-31-910-7268

E-mail: medipark@hanmail.net

(c) This is an Open Access article distributed under the terms of the Creative Commons Attribution Non-Commercial License (http://creativecommons.org/licenses/by$\mathrm{nc} / 3.0$ ) which permits unrestricted non-commercial use, distribution, and reproduction in any medium, provided the original work is properly cited. increased cardiovascular-disease-related morbidity and mortality, and decreased quality of life. ${ }^{4}$ In addition, the DS-14 score is used as a prognostic factor and a determinant of health status. ${ }^{5}$ Type-D personality was also associated with hypercholesterolemia in patients with myocardial infarction. ${ }^{6}$ Moreover, it has been associated with poor medication compliance in patients with some heart diseases.

It has been shown that the type- $\mathrm{D}$ personality is associated with certain psychiatric symptoms, including anxiety, depressive symptoms, and post-traumatic-stress disorder. ${ }^{8}$ In addition, both social and general anxiety were observed in general population with type-D personality. ${ }^{9}$ It was also reported that each type D subscale (NA and SI) was related to cortisol reactivity to stress. ${ }^{10}$ Recently, it was found that type-D personality was related to depression in parents of children with leukemia. ${ }^{11}$

Michal et al., ${ }^{12}$ in a study of a general German population, were the first to find an association between the type-D personality and suicidal ideation. However, no previous studies have investigated the potential link between the type-D per- 
sonality and suicidality including suicide attempt and suicial ideation in patients with major depressive disorder (MDD).

The present study tested the hypothesis that depressed patients with type-D personality are associated with increased suicidality. Thus, the aim of this study was to clarify the relationship between type-D personality and suicidality, including the history of suicide attempt, and suicidal ideation in patients with MDD.

\section{METHODS}

Eighty-six outpatients aged between 18 and 65 years who met the Diagnostic and Statistical Manual of Mental Disorders (DSM-IV) text revision criteria for MDD were recruited from Ilsan Paik Hospital from 2011 to 2013. The MDD diagnosis was determined in all subjects by trained psychiatrists. Subjects who had psychotic symptoms, any additional mental disorders on axis I or II of the DSM-IV, or major medical and neurological disorders were excluded in order to reduce possible bias. None of the subjects had a history of hypomanic or manic episodes.

The subjects were stratified into two subgroups according to the presence of type-D personality (using DS-14) and history of suicide attempt (yes vs. no) (by interview) and compared to each other. All demographic and clinical variables were measured before the beginning of the medication. Depression severity was evaluated using the Hamilton Depression Rating Scale (HAMD). ${ }^{10}$ Furthermore, the DS- $14,{ }^{3}$ the Beck Hopelessness Scale (BHS), ${ }^{7}$ the Barratt Impulsiveness Scale (BIS), ${ }^{11}$ the Hamilton Anxiety Scale (HAMA), ${ }^{9}$ and the Beck Scale for Suicidal Ideation (BSS) ${ }^{6}$ were applied. Validation studies of the Korean versions of all of these scales, with the exception of HAMA, have confirmed their validity and reliability as good psychometric tools. ${ }^{13-16}$ The study protocol was approved by the ethics committee of Inje University Ilsan Paik Hospital, and written informed consent to participate was obtained from all patients before beginning the investigation. Statistical analyses were performed using the statistical analysis system (SAS) version 9.3 and Intelligent Statistical Analysis Tool (SALT) version 2.5 software packages, and all results are reported as mean $\pm S D$ values. The demographic and clinical variables of the two subgroups were compared using Student's t-test, the chi-square test, correlation analysis (Pearson's correlation), and multiple linear logistic regression. All tests were two tailed, and group differences were tested at the $\mathrm{p}<$ 0.05 level.

\section{RESULTS}

In the present sample of 86 subjects with MDD, the age and HAMD score were $40.15 \pm 14.02$ years (mean \pm SD) and 17.77 \pm 4.77 , respectively. The prevalence of type-D personality in these subjects with MDD was $75.6 \%$.

The subjects were divided into two groups according to the DS-14-confirmed presence or absence of type-D personality, and several variables were compared between these groups (Table 1). The subjects were also divided into two groups according to whether or not they had a history of suicide attempt, with group comparisons also being performed (Table 2).

The BSS, BHS, and BIS scores were higher for the group with type-D personality than for the group without this personality $(\mathrm{p}=0.004,0.01$, and 0.003 , respectively) (Table 1 ). However, the HAMD and HAMA scores did not differ between these groups (Table 1).

In addition, the BSS, BHS, and SI scores were higher for the group with a history of suicide attempt than for the group without this history ( $\mathrm{p}=0.0000004,0.003$, and 0.033 , respectively) (Table 2). Although there was a tendency for the total DS-14 score to be higher in the group with a history of suicide attempt, the difference did not reach statistical significance $(\mathrm{p}=$ 0.064) (Table 2). The HAMD, HAMA, BIS, and NA scores did not differ significantly between these two groups. There were

Table 1. Comparison of demographic and clinical variables between groups with and without type-D personality

\begin{tabular}{lccc}
\hline \multicolumn{1}{c}{ Variable } & Group without type-D personality $(\mathrm{N}=21)$ & Group with type-D personality $(\mathrm{N}=65)$ & $\mathrm{p}$ \\
\hline Age, years & $48.05 \pm 9.53$ & $37.65 \pm 14.23$ & $0.0024^{*}$ \\
Sex $(\mathrm{M} / \mathrm{F})$ & $3 / 18$ & $17 / 48$ & $0.26^{\dagger}$ \\
Total DS-14 score & $19.38 \pm 7.38$ & $37.89 \pm 8.74$ & $<0.01^{*}$ \\
Total HAMD score & $17.52 \pm 5.09$ & $17.82 \pm 4.71$ & 0.81 \\
Total HAMA score & $19.33 \pm 6.13$ & $20.57 \pm 5.03$ & 0.40 \\
Total BSS score & $7.29 \pm 6.46$ & $13.8 \pm 9.36$ & $0.004^{*}$ \\
Total BHS score & $7.86 \pm 5.58$ & $11.78 \pm 6.04$ & $0.01^{*}$ \\
Total BIS score & $69.05 \pm 11.84$ & $79.66 \pm 14.40$ & $0.003^{*}$ \\
\hline
\end{tabular}

Except where stated otherwise, data are mean \pm SD values. *statistically significant difference at $\mathrm{p}<0.05$, ${ }^{\dagger}$ goodness-of-fit test. DS-14: type-D Personality Scale-14, HAMD: Hamilton Depression Rating Scale, HAMA: Hamilton Anxiety Scale, BSS: Beck Scale for Suicidal Ideation, BHS: Beck Hopelessness Scale, BIS: Barratt Impulsiveness Scale, F: female, M: male 
Table 2. Comparison of demographic and clinical variables between groups with and without a history of suicide attempt

\begin{tabular}{lccc}
\hline \multicolumn{1}{c}{ Variable } & Group without a history of suicide attempt $(\mathrm{N}=54)$ & Group with a history of suicide attempt $(\mathrm{N}=32)$ & $\mathrm{p}$ \\
\hline Age, years & $41.85 \pm 13.63$ & $37.38 \pm 14.19$ & 0.15 \\
Sex (M/F) & $15 / 40$ & $5 / 28$ & $0.2^{\dagger}$ \\
Total HAM-D score & $17.69 \pm 4.45$ & $17.84 \pm 5.35$ & 0.88 \\
Total HAM-A score & $19.61 \pm 5.42$ & $21.37 \pm 6.36$ & 0.18 \\
Total BSS score & $8.57 \pm 6.89$ & $18.34 \pm 9.3$ & $0.0000004^{*}$ \\
Total BHS score & $9.35 \pm 6.16$ & $13.31 \pm 5.33$ & $0.003^{*}$ \\
Total BIS score & $77.87 \pm 14.77$ & $75.66 \pm 14.07$ & 0.5 \\
Total DS-14 score & $31.59 \pm 10.73$ & $36.38 \pm 12.51$ & 0.064 \\
Total NA score & $17.81 \pm 5.87$ & $19.81 \pm 6.77$ & 0.15 \\
Total SI score & $14.15 \pm 6.87$ & $17.47 \pm 6.93$ & $0.033^{*}$ \\
\hline
\end{tabular}

Except where stated otherwise, data are mean \pm SD values. *statistically significant difference at $\mathrm{p}<0.05$, ${ }^{\dagger}$ goodness-of-fit test. DS-14: type-D Personality Scale-14, HAM-D: Hamilton Depression Rating Scale, HAM-A: Hamilton Anxiety Scale, BSS: Beck Scale for Suicidal Ideation, BHS: Beck Hopelessness Scale, BIS: Barratt Impulsiveness Scale, F: female, M: male, NA: negative affect subscale, SI: social inhibition subscale

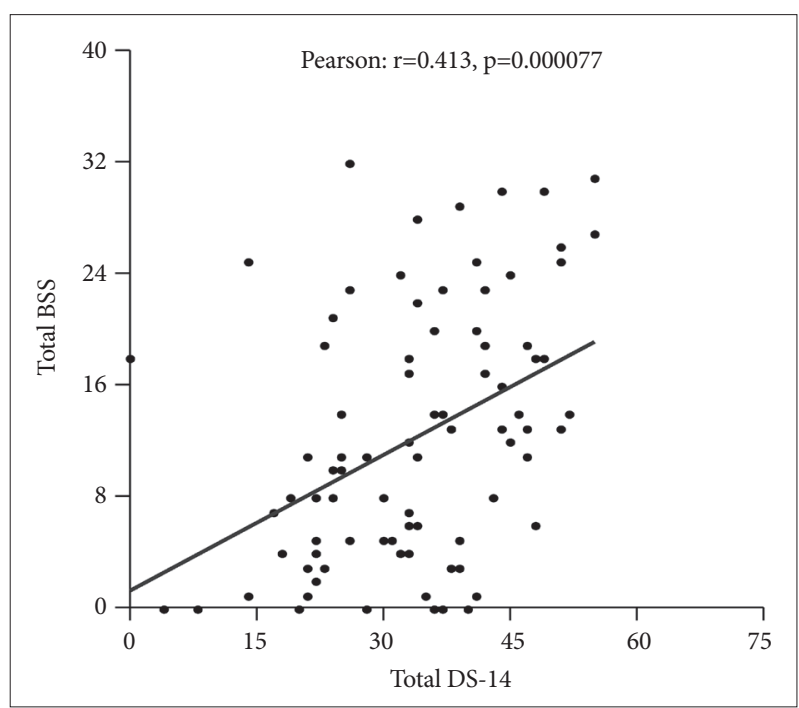

Figure 1. Correlation between total scores on the type-D Personality Scale-14 (DS-14) and Beck Scale for Suicidal Ideation (BSS).

positive correlations between the total DS-14 score and the total BSS, BHS, and BIS scores $(r=0.413$ and $\mathrm{p}=0.000077, \mathrm{r}=$ 0.404 and $\mathrm{p}=0.00012$, and $\mathrm{r}=0.245$ and $\mathrm{p}=0.024$, respectively) (Figure 1-3).

Multiple linear logistic regression analysis revealed significant relationships between the total BSS score and total BHS, NA, and a history of suicide attempt ( $\mathrm{p}=0.000002,0.022$, and 0.000006 , respectively) (Table 3).

\section{DISCUSSION}

The findings of this study demonstrate the existence of differences in suicidality between depressed patients with and without type-D personality. Namely, it appears that the type$\mathrm{D}$ personality is associated with suicidal ideation and a history of suicide attempt. No previous studies have investigated the

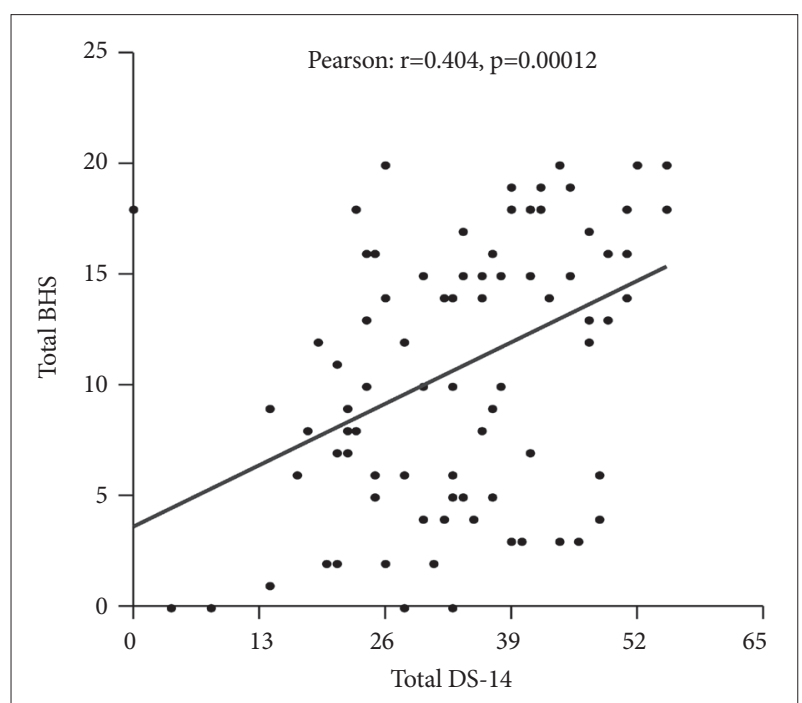

Figure 2. Correlation between total scores on the DS-14 and Beck Hopelessness Scale (BHS).

relationship between type-D personality and suicide attempt or suicidal ideation among patients with MDD.

It has been established that individuals with a type-D personality and cardiovascular disease present with more psychological problems and a worse outcome. ${ }^{4}$ For example, the type$\mathrm{D}$ personality was associated with increased symptoms of anxiety and depression in patients with cardiovascular disease, independent of sociodemographic and clinical risk factors. ${ }^{8}$ Similarly, type-D personality in cardiovascular patients was found to be associated with psychological distress and an unhealthy lifestyle. ${ }^{17}$ In addition, a recent meta-analysis found that the type-D personality was an independent correlate of impaired patient-reported physical and mental health status in various cardiovascular patient groups. ${ }^{18}$

Most studies of type-D personality have focused on cardiovascular patients. However, it was recently found that the risk 


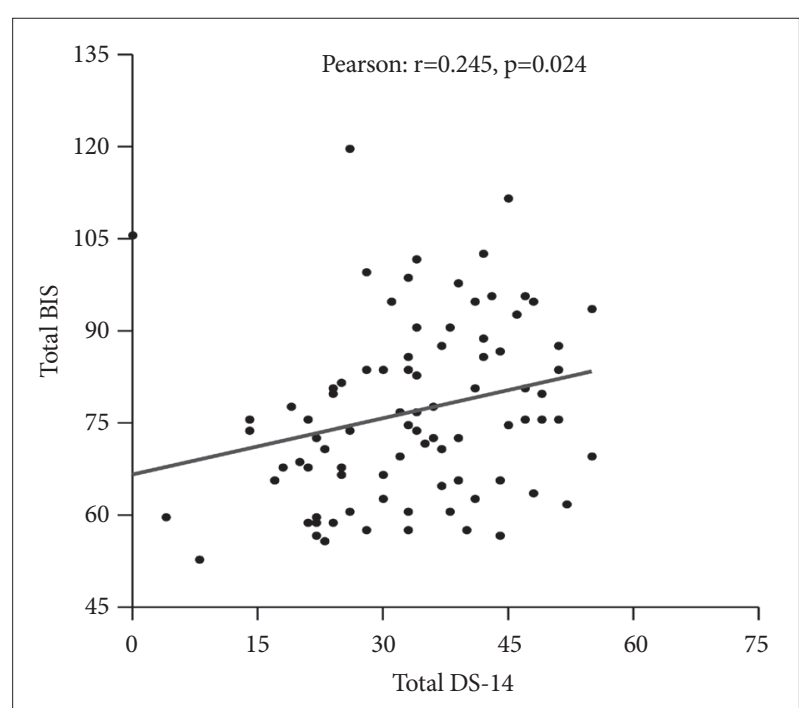

Figure 3. Correlation between total scores on the DS-14 and Barratt Impulsiveness Scale (BIS).

Table 3. Multiple linear logistic regression between suicidal ideation (total BSS score) and several variables

\begin{tabular}{lccl}
\hline \multicolumn{1}{c}{ Variable } & Coefficient & SE & \multicolumn{1}{c}{$\mathrm{p}$} \\
\hline Intercept & -4.86 & 4.006 & 0.229 \\
Total BIS score & 0.031 & 0.051 & 0.543 \\
Total BHS score & 0.596 & 0.131 & $0.000002^{*}$ \\
Total NA score & 0.638 & 0.273 & $0.022^{*}$ \\
Total SI score & 0.37 & 0.281 & 0.191 \\
Total DS-14 score & -0.348 & 0.254 & 0.174 \\
Suicide-attempt history & 6.455 & 1.521 & $0.000006^{*}$ \\
\hline
\end{tabular}

*statistically significant difference at $\mathrm{p}<0.05$. SE: standardized error, DS-14: type-D Personality Scale-14, BSS: Beck Scale for Suicidal Ideation, BHS: Beck Hopelessness Scale, BIS: Barratt Impulsiveness Scale, F: female, M: male, NA: negative affect subscale, SI: social inhibition subscale

of psychological distress was four to five fold higher in elderly individuals with type-D personality. ${ }^{19}$ In addition, both depression and type-D personality were found to be present in $36 \%$ of medical students at a medical school in West Bengal..$^{20}$ Furthermore, a link has been demonstrated between type-D personality and suicidal ideation in the general population in Germany. ${ }^{12}$ These findings are consistent with those of the present study. Despite the lack of significant differences in total HAMD and HAMA scores between groups with and without type-D personality, the total BSS and BHS scores did differ between these two groups (Table 1). This suggests that depressed patients with type-D personality are more vulnerable to suicidality than those without type-D personality, even when the MDD severity is identical. In addition, the total BSS, BHS, and SI scores were higher in the group with a history of suicide attempt than the group without this history, despite the total HAMD and HAMA scores not differing between these two groups (Table 2). Thus, it appears that previous suicide attempts can increase the possibility of reattempts, since the total BSS and BHS scores were also higher in the group with a history of suicide attempt. Intriguingly, the SI score (a subscale of DS-14) was higher in the group with a history of suicide attempt than in the group without this history. Thus, the typeD personality appears to be related to suicidality in patients with MDD.

There were positive correlations between the total DS-14 score and the total BSS, BHS, and BIS scores (Figure 1-3). These results also indicate the existence of correlations between type-D personality and suicidality and impulsivity. Furthermore, multiple linear logistic regression analysis revealed that suicidal ideation (BSS) is related to hopelessness (BHS), typeD personality (NA), and previous suicide attempts (Table 3).

The small sample in this study limits the generalizability of its results. However, notwithstanding this limitation, the present findings have revealed differences between depressed patients according to the presence of type-D personality with respect to BSS, BHS, and BIS scores. In addition, the age was younger for the group with type-D personality than for the group without this personality. It is possible that the age may affect total DS-14 score. However, multiple linear regression analysis revealed that the association between total DS-14 score and age was not statistically significant $(\mathrm{p}=0.28)$. More studies involving larger samples and evaluating differences between subjects with and without the type-D personality are needed.

\section{Acknowledgments}

This study was supported by a grant from National Research Foundation of Korea (NRF), funded by Ministry of Education and Science Technology (MEST) (2011-0010562). The authors would like to thank Jo BW for her assistance with data collection.

\section{REFERENCES}

1. Denollet J. Personality and coronary heart disease: the type-D scale-16 (DS16). Ann Behav Med 1998;20:209-215.

2. Mols F, Denollet J. Type D personality among noncardiovascular patient populations: a systematic review. Gen Hosp Psychiatry 2010;32: 66-72.

3. Denollet J. DS14: standard assessment of negative affectivity, social inhibition, and Type D personality. Psychosom Med 2005;67:89-97.

4. Denollet J, Sys SU, Brutsaert DL. Personality and mortality after myocardial infarction. Psychosom Med 1995;57:582-591.

5. Pedersen SS, Denollet J, Ong AT, Sonnenschein K, Erdman RA, Serruys $\mathrm{PW}$, et al. Adverse clinical events in patients treated with sirolimus-eluting stents: the impact of Type D personality. Eur J Cardiovasc Prev Rehabil 2007;14:135-140.

6. Bagherian-Sararoudi R, Sanei H, Attari A, Afshar H. Type D personality is associated with hyperlipidemia in patients with myocardial infarction. J Res Med Sci 2012;17:543-547.

7. Wu JR, Moser DK. Type D Personality Predicts Poor Medication Adherence in Patients with Heart Failure in the USA. Int J Behav Med 2013 inpress. 
8. Spindler H, Kruse C, Zwisler AD, Pedersen SS. Increased anxiety and depression in Danish cardiac patients with a type D personality: crossvalidation of the Type D Scale (DS14). Int J Behav Med 2009;16:98-107.

9. Kupper N, Denollet J. Type D Personality Is Associated with Social Anxiety in the General Population. Int J Behav Med 2013 inpress.

10. Sher L. Type D personality: the heart, stress, and cortisol. QJM 2005; 98:323-329.

11. Chen J, Liu Y, Cai Q, Liu Y, Wang T, Wang J, et al. Depression in parents of children with leukemia in southern China accompanied by the prevalence of type D personality. Support Care Cancer 2014;22:1277-1286.

12. Michal M, Wiltink J, Till Y, Wild PS, Munzel T, Blankenberg S, et al. Type-D personality and depersonalization are associated with suicidal ideation in the German general population aged 35-74: results from the Gutenberg Heart Study. J Affect Disord 2010;125:227-233.

13. Park KB, Shin MS. Perceived stress and suicidal ideation of high school students. Korean J Clin Psychol 1991;10:298-314.

14. Shin MS, Park KB, Oh KJ, Kim JS. A study of suicidal ideation among high school students: the structural relation among depression, hopelessness, and suicidal ideation. Korean J Clin Psychol 1990;9:1-19.
15. Yi JS, Bae SO, Ahn YM, Park DB, Noh KS, Shin HK, et al. Validity and reliability of the Korean version of the Hamilton Depression Rating Scale(K-HDRS). J Korean Neuropsychiatr Assoc 2005;44:456-465.

16. Chung YO, Lee CW. A study of factor structures of the Barratt impulsiveness scale in Korean university students. Korean J Clin Psychol 1997; 16:117-129.

17. Svansdottir E, van den Broek KC, Karlsson HD, Gudnason T, Denollet J. Type D personality is associated with impaired psychological status and unhealthy lifestyle in Icelandic cardiac patients: a cross-sectional study. BMC Public Health 2012;12:42.

18. Versteeg H, Spek V, Pedersen SS, Denollet J. Type D personality and health status in cardiovascular disease populations: a meta-analysis of prospective studies. Eur J Prev Cardiol 2012;19:1373-1380.

19. Kasai Y, Suzuki E, Iwase T, Doi H, Takao S. Type D Personality Is Associated with Psychological Distress and Poor Self-Rated Health among the Elderly: A Population-Based Study in Japan. PLoS One 2013;8: e77918.

20. Gupta S, Basak P. Depression and type D personality among undergraduate medical students. Indian J Psychiatry 2013; 55: 287-289. 